Analytical Methods in Environmental Chemistry Journal Vol 2 (2019) 59-70

Research Article, Issue 4
Analytical Methods in Environmental Chemistry Journal
Journal home page: www.amecj.com/ir

\title{
Removal of ethylbenzene from air by graphene quantum dots and multi wall carbon nanotubes in present of UV radiation
}

\author{
Maling Gou ${ }^{\mathrm{a}, \mathrm{b}}$ and Baharak Bahrami Yarahmadi ${ }^{\mathrm{c}, *}$ \\ ${ }^{a}$ State Key Laboratory of Biotherapy and Cancer Center, West China Hospital, Sichuan University and Collaborative Innovation Center for Biotherapy, Chengdu \\ 610041, China \\ ${ }^{b}$ Department of Thoracic Oncology, Cancer Center, West China Hospital, Medical School, Sichuan University, Chengdu 610041, China \\ C,* Occupational Health Engineering Department, School of Public Health, Kerman University of Medical Sciences, Kerman, Iran
}

\section{A R T I C L E I N F O:}

Received 12 Sep 2019

Revised form 15 Nov 2019

Accepted 5 Dec 2019

Available online 28 Dec 2019

\section{Keywords:}

Graphene quantum dots, Multi wall carbon nanotubes, Ethylbenzene,

Air removal, UV-radiation, Solid gas removal

\begin{abstract}
A B S T R A C T
Luminescent graphene quantum dots (GQDs) and multi wall carbon nanotubes (MWCNTs) as photocatalytic sorbent based on was used for removal of toxic ethylbenzene from air in present of UV-radiation. A novel method based on solid gas removal (SGR) based on GQDs and MWCNTs as an efficient adsorbent was used for ethylbenzene removal from air in Robson quartz tubes (RGT). After synthesized and purified of GQDs and MWCNTs, a system was designed for generation of ethylbenzene in air with difference concentrations, and then the mixture was moved to quartz tubes with UV radiation in optimized conditions. The ethylbenzene in air was absorbed on the $25 \mathrm{mg}$ of GQDs or MWCNTs, desorbed from sorbent at $146^{\circ} \mathrm{C}$ and determined by GC-FID. The main parameters such as, temperature, ethylbenzene concentration, amount of GQDs / MWCNTs and flow rate were studied and optimized. The recovery of ethylbenzene removal from air (more than 95\%) and absorption capacity of adsorbent (186.4 $\mathrm{mg} \mathrm{g}^{-1}$ ) were achieved in present of UV radiation at room temperature by GQDs. The flow rate and temperature were obtained at $300 \mathrm{~mL} \mathrm{~min}^{-1}$ and less than $42{ }^{\circ} \mathrm{C}$, respectively. Based on results, the special surface area and favorite porosity of GQDs caused to efficient removal of ethylbenzene from air in present of UV as compared to other carbon compounds such as MWCNTs, and graphene.
\end{abstract}

\section{Introduction}

One of the most important issues facing human beings today and even endangering their health is air pollution. Volatile organic compounds (VOCs) are one of the most important pollutants, and these compounds are listed as toxic $[1,2]$. Global warming, ozone depletion, photochemical smog, and contributor of haze is the effect of this material $[3,4]$. The boiling point range of volatile organic compounds is from 50 to $250{ }^{\circ} \mathrm{C}$ and because of

\footnotetext{
*Corresponding Author: Baharak Bahrami Yarahmadi Email: baharakb72@gmail.com

DOI: https://doi.org/10.24200/amecj.v2.i04.82
}

high vapor pressure creates a notable amount of the molecules to evaporate and release in the air $[5,6]$. Their health effects on humans are very important, these compounds can irritate the respiratory system and eyes, cause headaches and nausea, damage the kidneys, liver, the central nervous system and even in chronic exposure cause cancer [7-10]. Some of the major industries producing volatile organic compounds include petroleum refineries, chemical industries, automotive industries, paint industry, pharmaceuticals, cable and wire industries, printing, aerospace, textile, etc. [1, 11]. BTEX (Benzene, toluene, ethylbenzene, and xylene) are the most common VOCs and usually used in the 
petrochemical industry and as reagents for the synthesis of multiple C-based products [11-13]. Among BTEX, ethylbenzene is mainly used in the manufacture of styrene. The release of Ethylbenzene into the air could be carcinogenic, cause secondary aerosol and photochemical smog. Ethylbenzene is a colorless liquid that smells like gasoline. The odor threshold for ethylbenzene is 2.3 parts per million (ppm). The chemical formula for ethylbenzene is $\mathrm{C}_{8} \mathrm{H}_{10}$, and the molecular weight is $106.16 \mathrm{~g} \mathrm{~mol}^{-1}$. The vapor pressure for ethylbenzene is 9.53 $\mathrm{mm} \mathrm{Hg}$ at $25{ }^{\circ} \mathrm{C}$, and its octanol/water partition coefficient is 3.13. In petrochemical factories, BTEX and mercury vapor released in air and can be absorbed in humans via the inhalation and dermal routes of exposure. So determination BTEX and mercury in air, water and human matrixes is very important [14-17]. Previous study reported the carcinogenic effects of ethylbenzene in humans. EPA has classified ethylbenzene as a Group D, not classifiable as to human carcinogenicity. ACGIH recommends a TLV-TWA of $100 \mathrm{ppm}$ and STEL/C of $125 \mathrm{ppm}$ for ethylbenzene based on irritation and central nervous system effects [18-20]. Acute (short-term) exposure to ethylbenzene in humans results in respiratory effects, such as throat irritation and chest constriction, irritation of the eyes, and neurological effects such as dizziness. Chronic (long-term) exposure to ethylbenzene by inhalation in humans has shown conflicting results regarding its effects on the blood. Animal studies have reported effects on the blood, liver, and kidneys from chronic inhalation exposure to ethylbenzene [2123]. There are many successful techniques which have been developed and applied to control the VOCs emission, such as condensation, membrane, absorption, adsorption, thermal combustion, catalytic, photocatalytic oxidation, non-thermal plasma, and biofiltration [24-27]. Photocatalytic oxidation (PCO) as the most current generation of air cleaning technology has a magnificent potential to eliminate vaporous pollutants even at low concentrations [28]. Exceptional features of this method are operating at ambient temperature without notable energy supply, environmentally friendly final products $\left(\mathrm{CO}_{2}\right.$ and $\left.\mathrm{H}_{2} \mathrm{O}\right)$, and applicable for various pollutants [29]. PCO implemented using photocatalyst, ultraviolet (UV) light and oxygen to decay chemical pollutants[30]. Numerous researchers have reviewed the materials for the removal of VOCs [27, 31]. Most sources have been reviewed based on a particular kind of material, such as $\mathrm{TiO}_{2}$ [32], graphene-based materials [33], zinc indium sulfide [34] and silicananosphere-based materials, etc., or concentrating on the catalytic oxidation processes in a specific condition such as low-temperature, visible light, or based on a review of the aspect of different VOCs[35].

In this study, a novel analytical method based on UV- GQDs or UV-MWCNTs was used for ethylbenzene removal from air by SGR technology. All of important parameter for photocatalytic process were optimized and the results validated by spiking standard concentration of ethylbenzene to real samples. The mixture of ethylbenzene vapor in air was generated and storage in polyethylene bags and its concentration determined by GCMS before moved to quartz tubes. The removal efficiencies were calculated in UV- GQDs, GQDs and $\mathrm{MWCNT}_{\mathrm{S}}$ by SGR procedure.

\section{Experimental}

\subsection{Gas Chromatography (GC-FID)}

The gas chromatography with flame ionization detector (GC-FID) based on air sample loop injection was used for ethylbenzene determination in gas phase (Agilent GC, 7890A, FID, Netherland). Before injection to GC-FID, Slide the plunger carrier down and tighten. An air sample introduced into the carrier gas by sampling valves which was used to sample gases or liquids. Air sampling bags were used by valve and septum port (Tedlar, Germany). GC with a split injector $\left(200^{\circ} \mathrm{C}\right)$, flame ionization detector $\left(250^{\circ} \mathrm{C}\right)$, and a column with methylsiloxane was used. The oven temperature was adjusted from $25^{\circ} \mathrm{C}$ to $250^{\circ} \mathrm{C}$ which was held for $15 \mathrm{~min}$. The carrier gas of hydrogen with flow rate of $1.2 \mathrm{~mL} \mathrm{~min}^{-1}$ were tuned. For batch system, the vials (PTFE) with air-tight cap (parker) were 
prepared. TGS 2180 (China) and Dräger 3500 (Lübeck, Germany) as gas detectors was used for determination of $\mathrm{H}_{2} \mathrm{O}$ vapor and $\mathrm{O}_{2}$ in air. The ethylbenzene was evaporated and mixed with purified air at $135^{\circ} \mathrm{C}$ in chamber.

\subsection{Reagents}

The ultra-pure chemicals were purchased from Merck and Sigma Aldrich (Germany). The Deionized Distilled water (DDW) was prepared by (Millipore, CAS 7732-18-5). The standard of ethylbenzene $\left(\mathrm{C}_{6} \mathrm{H}_{5} \mathrm{C}_{2} \mathrm{H}_{5}\right)$ was generated with ultra-pure air in chamber. The accuracy and precision of the pilot was investigated by injecting a concentration of ethylbenzene in chamber and determination of ethylbenzene in air bags by GC-MS before moved to RGT which was filled with GQDs or MWCNTs. The high purity of ethylbenzene was purchased from Merck (CAS N: 100-41-4, EC N: 202-849-4, Germany) and the calibration solutions of $0.1,0.2,0.5,1.0,1.5$ and 2.0 $\%(\mathrm{v} / \mathrm{v})$ were prepared. The GQDs and MWCNTs were synthesized by RIPI.

\subsection{Pilot of gas generation}

By pilot design, the purified air was prepared based on HEPA filter and activated carbon (HEPAAC) with electro air cleaner (EAC, Canada). The HEPA-AC removed VOCs and the particles dust from 200 to $300 \mathrm{~nm}$. The pure air passed through

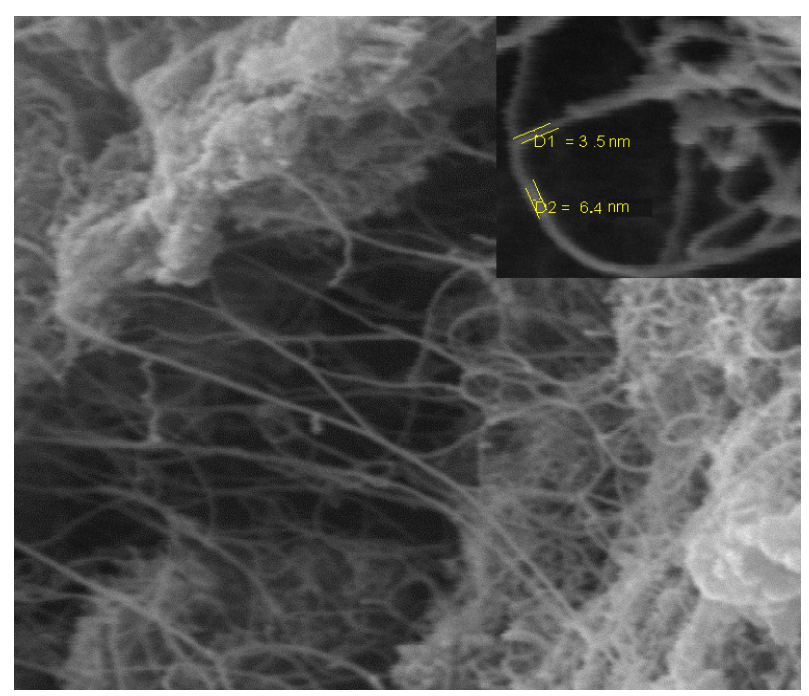

Fig. 1a. SEM of MWCNTs connection of PVC tubes and storage in 1-5 liter of bulk bag. After adjusting of $\mathrm{H}_{2} \mathrm{O}$, the mixture moved to GQDs or MWCNTs in optimized flow rate and temperature. All of lines and bags were covered with heating jackets capable of controlling the temperature up to $70{ }^{\circ} \mathrm{C}$ to prevent condensing.

\subsection{Synthesis of LGQDs and MWCNTS}

High-purity MWCNTs were synthesized by use of camphor, an environment-friendly hydrocarbon as a carbon source using chemical vapor deposition (CVD) method on $\mathrm{Co}-\mathrm{Mo} / \mathrm{MgO}$ Nano-catalysts [36]. We prepared GO from graphite adopting a modified Hummers' method [37, 38]. The GO was used for synthesizing of GQDs by Dong et al. Firstly, the amount of GO was refluxed with $\mathrm{HNO} 3$ $(10 \mathrm{M})$ at $120^{\circ} \mathrm{C}$ for one day. When the reaction was completed the color of solvent darkened. Then, the suspension was centrifuged for $15 \mathrm{~min}$ after being cooled at $25^{\circ} \mathrm{C}$. The suspension was collected after washing of product with DW and then centrifuged. Secondly, the obtained GO was dispersed in 20 $\mathrm{mL}$ DW, heated hydrothermally in a Teflon-lined stainless steel at $220^{\circ} \mathrm{C}$ for 10 hours and centrifuged (3500) for 20 min (brown color). So, GQDs were obtained in this procedure by green fluorescence under $365 \mathrm{~nm}$ UV light irradiation [39].

\subsection{Characteristics}

After hydrothermal method for synthesis nano

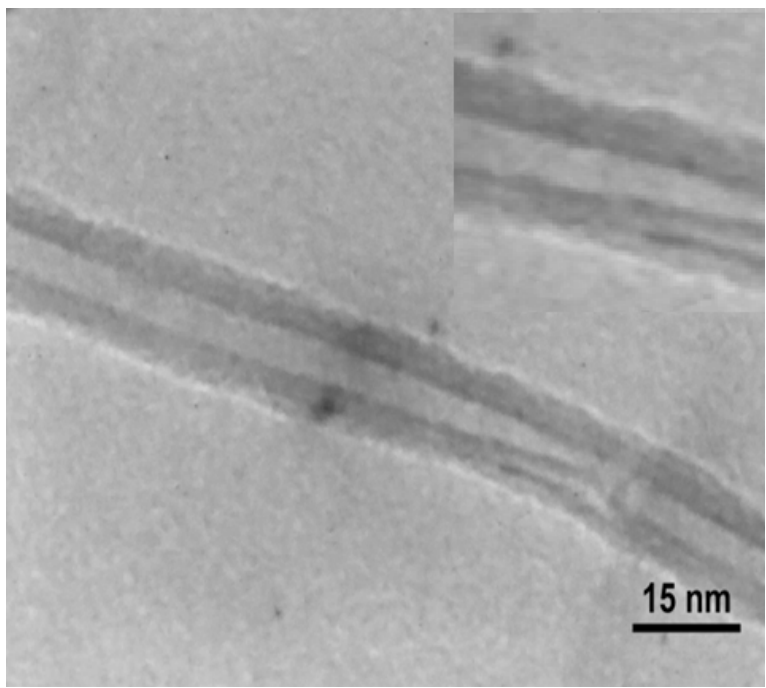

Fig. 1b. TEM of MWCNTs 


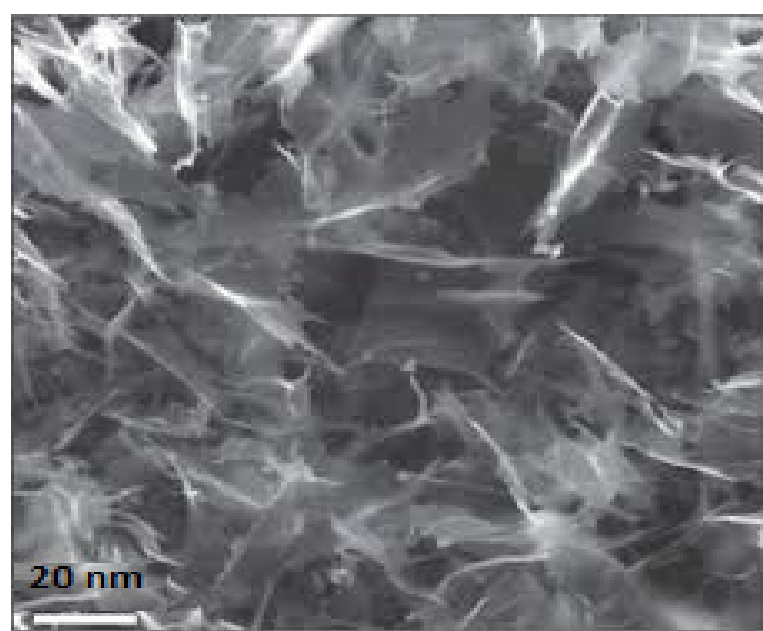

Fig. 2a. SEM of GQDs

materials, the SEM and TEM images of the MWCNTs and GQDs were shown in Figure 1 (a, b) and 2(a,b). The surface area and pore size of GQDs and MWCNTs based on nitrogen adsorption was evaluated by Brunauer-Emmett-Teller (BET) method. The surface area and porosity of the MWCNTs and GQDs, before and after heat treatment were similar values. Raman spectra of GQDs and MWCNTs show the G and D bands that are characteristic for carbon structures. Raman spectra show quality of nanostructure which was deepened on $I_{G} / I_{D}$ (Fig. 3). The pore size, length, BET surface area and textural properties of GQDs and MWCNTs were shown in Table 1 and 2.

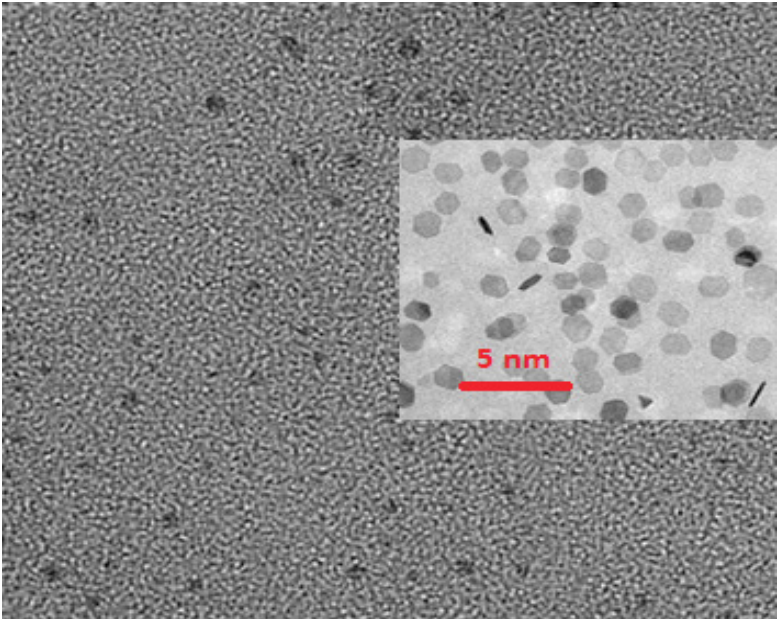

Fig. 2b. TEM of GQDs

\subsection{Removal Procedure}

The $25 \mathrm{mg}$ of different GQDs and MWCNTs, was used as sorbents for removal of ethylbenzene from air in optimized conditions (flow rate 300 $\left.\mathrm{mL} \min ^{-1}, 42{ }^{\circ} \mathrm{C}\right)$. The different concentration of ethylbenzene in air (bulk bag) was passed through the GQDs and MWCNTs sorbents. After efficient adsorption in present of UV radiation, the ethylbenzene concentration in air was determined by GC-FID. Also, the removal efficiency calculated after desorption of ethylbenzene from GQDs and MWCNTs by thermal accessory at $150{ }^{\circ} \mathrm{C}$. For sample blank, $1 \mathrm{~mL}$ of air in bulk bag was injected

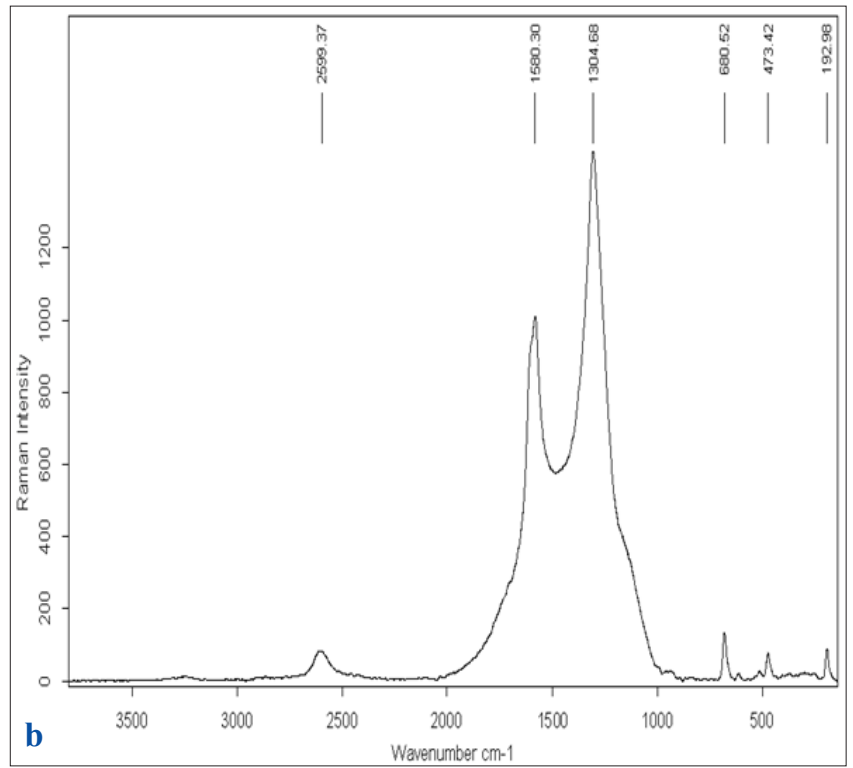

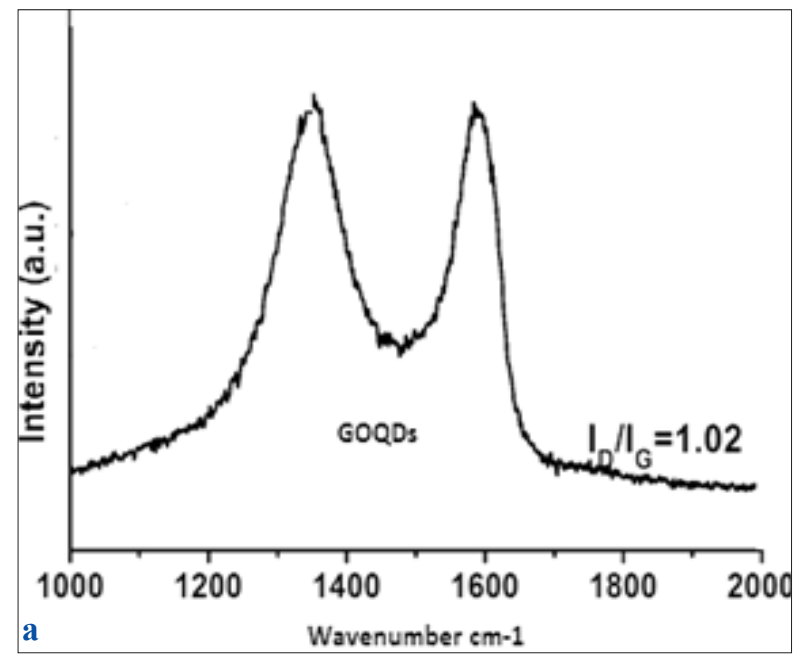

Fig. 3. Raman spectroscopy of a) GQDs and b) MWCNTs 
Table 1. Textural properties of GQDs and MWCNTs

\begin{tabular}{lcccccc}
\hline Carbon & $\mathbf{S}_{\text {BET }}{ }^{\mathbf{a}}\left(\mathbf{m}^{2} / \mathbf{g}\right)$ & $\mathbf{d}_{\mathbf{s p}}{ }^{\mathbf{b}}(\mathbf{n m})$ & $\mathbf{d}_{\mathrm{lp}}{ }^{\mathbf{c}}(\mathbf{n m})$ & $\mathbf{V}_{\mathbf{s p}}{ }^{\mathbf{d}}\left(\mathbf{c m}^{3} / \mathbf{g}\right)$ & $\mathbf{V}_{\mathrm{lp}}{ }^{\mathbf{e}}\left(\mathbf{c m}^{\mathbf{3} / \mathbf{g})}\right.$ & $\mathbf{P A}(\mathbf{A})$ \\
\hline MWCNT & 375 & 5.54 & 15.08 & 0.51 & 1.04 & 117.52 \\
GQDs & 343 & 4.65 & 14.17 & 0.53 & 0.89 & 101.18 \\
\hline
\end{tabular}

${ }^{\mathrm{a} B E T}$ specific surface area, ${ }^{\mathrm{b}}$ diameter of small pores, ${ }^{\mathrm{c}}$ diameter of large pores, ${ }^{\mathrm{d}}$ Volume of small pores, ${ }^{e}$ Volume of large pores, pore Diameter (PA)

Table 2. Pore size, length and BET surface area of GQDs and MWCNTs

\begin{tabular}{lcccc}
\hline Carbon & Diameter $(\mathbf{n m})$ & Length $(\mathbf{u m})$ & ${ }^{*} \mathbf{I}_{\mathbf{G}} / \mathbf{I}_{\mathbf{D}}$ & Surface Area $\left(\mathbf{m}^{2} / \mathbf{g r}\right)$ \\
\hline MWCNT & $4-20$ & $8-14$ & 0.77 & 375 \\
GQDs & $3-15$ & $8-12$ & 0.68 & 346 \\
\hline
\end{tabular}

*(IG/ID): LG band originates from ordered, well-graphitized carbon, D band is the disorder-activated band

to injector of GC-FID by Hamilton syringes and the concentration of ethylbenzene was determined by GC-FID and GC-MS (Agilent 7890A, USA). So, SGR procedure based on GQDs can be efficiently removed ethylbenzene from air.

\section{Results and Discussion}

\section{1. Optimizing of parameters}

In optimized conditions, the adsorption capacity of ethylbenzene in an air is the amount of adsorbate ethylbenzene ( $\mathrm{mg}$ ) on GQDs sorbent $(\mathrm{g})$. The removal efficiency of GQDs is the ratio of removed ethylbenzene to initial ethylbenzene concentration in air. The removal efficiency and adsorption capacity are depended on the important parameters such as; kind of sorbent, size of nanoparticles, temperature, flow rate, ethylbenzene concentration and humidity which were optimized. The effect of ethylbenzene concentration was investigated by SGR method from 1.0 to $100 \mathrm{ppm}$. The results showed us, high concentration of ethylbenzene, based on the GQDs was saturated early graphene dot sites. In optimized conditions, the ethylbenzene concentration for $25 \mathrm{mg}$ of GQDs and MWCNTs was achieved, $4.66 \mathrm{ppm}$ and $2.54 \mathrm{ppm}$, respectively in $25^{\circ} \mathrm{C}$ (Fig. 4). So, the absorption capacity was achieved $186.4 \mathrm{mg} \mathrm{g}^{-1}$ and $102.4 \mathrm{mg} \mathrm{g}^{-1}$, respectively (Fig. 5). For ethylbenzene removal from air, the effects of humidity on removal efficiency of GQDs and MWCNTs were studied between 10\% - 70\%.

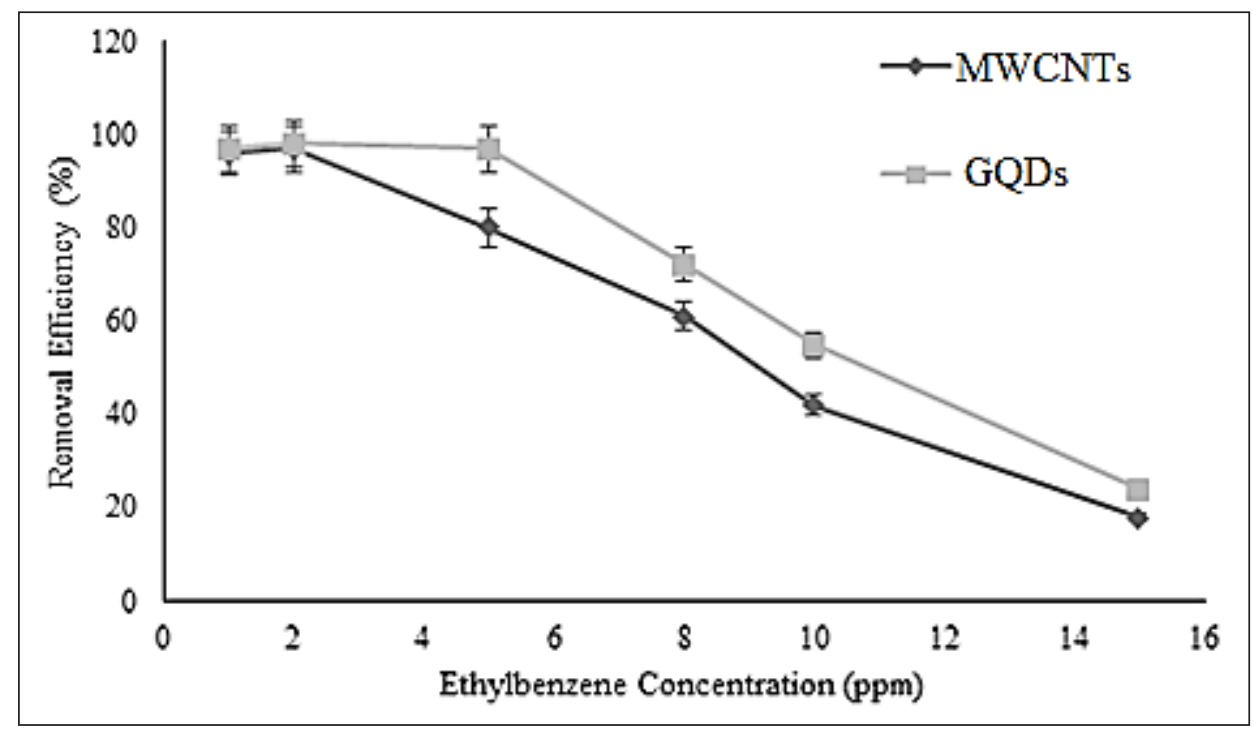

Fig. 4. The effect of ethylbenzene concentration on air removal 


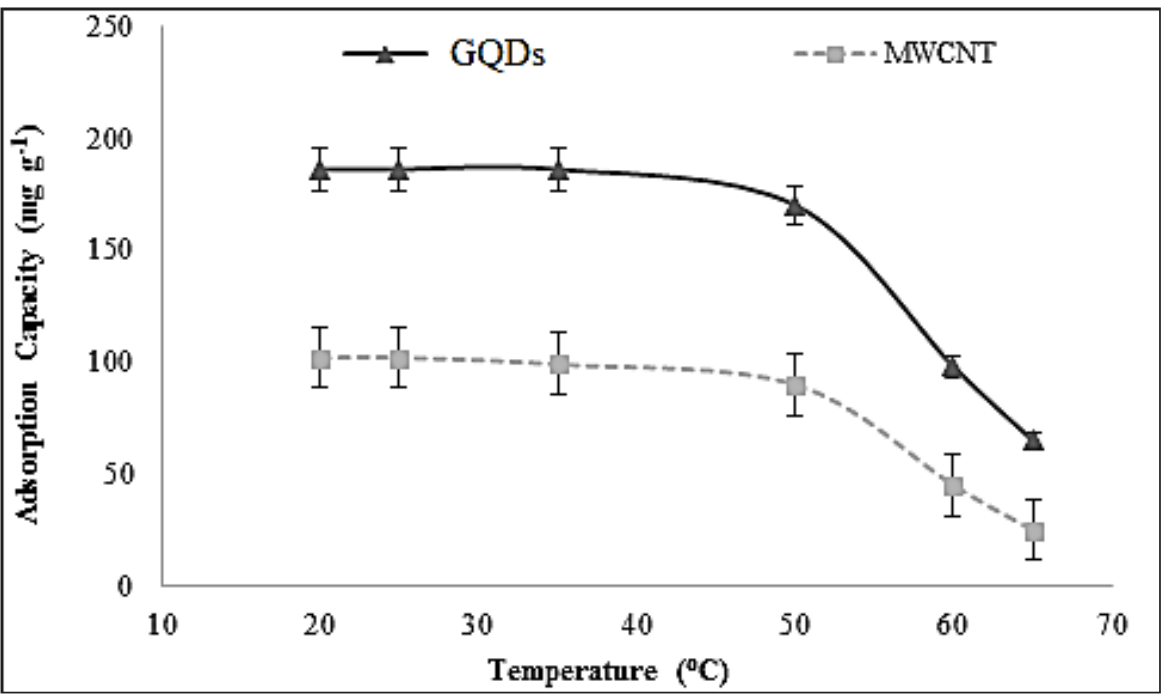

Fig. 5. The effect of temperature on absorption capacity for ethylbenzene removal from air by GQDs

The results showed, by increasing of humidity up to $10 \%$, the removal efficiency wasn't decreased. The temperature has effected on adsorption capacity and recovery of GQDs for ethylbenzene removal from air. The effect of temperature was studied between $25-150{ }^{\circ} \mathrm{C}$. The results showed us, the absorption efficiency of ethylbenzene by GQDs was achieved under $420{ }^{\circ} \mathrm{C}$ and desorption was obtained at $146^{\circ} \mathrm{C}$ (Fig. 6). In optimized flow rate value, the maximum recovery was happened by GQDs by SGR procedure. So, the effect of different flow

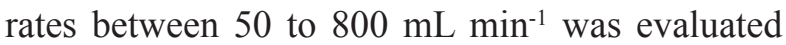

based on GQDs for ethylbenzene removal from air. The results showed, the recovery of removal was decreased in more than $350 \mathrm{~mL} \mathrm{~min}^{-1}$. Therefore,

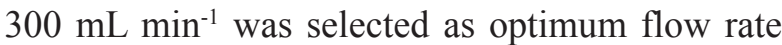
(Fig. 7). The inside of quartz tubes was filled with GQDs and MWCNTs as a sorbent for ethylbenzene removal from air. Diameter and length of quartz tubes and physical and chemical properties of GQDs and MWCNTs is important factor for adsorption efficiency of ethylbenzene which must be optimized. Based on results, $0.3 \mathrm{~cm}$ of diameter and $5 \mathrm{~cm}$ of length selected as optimum column for

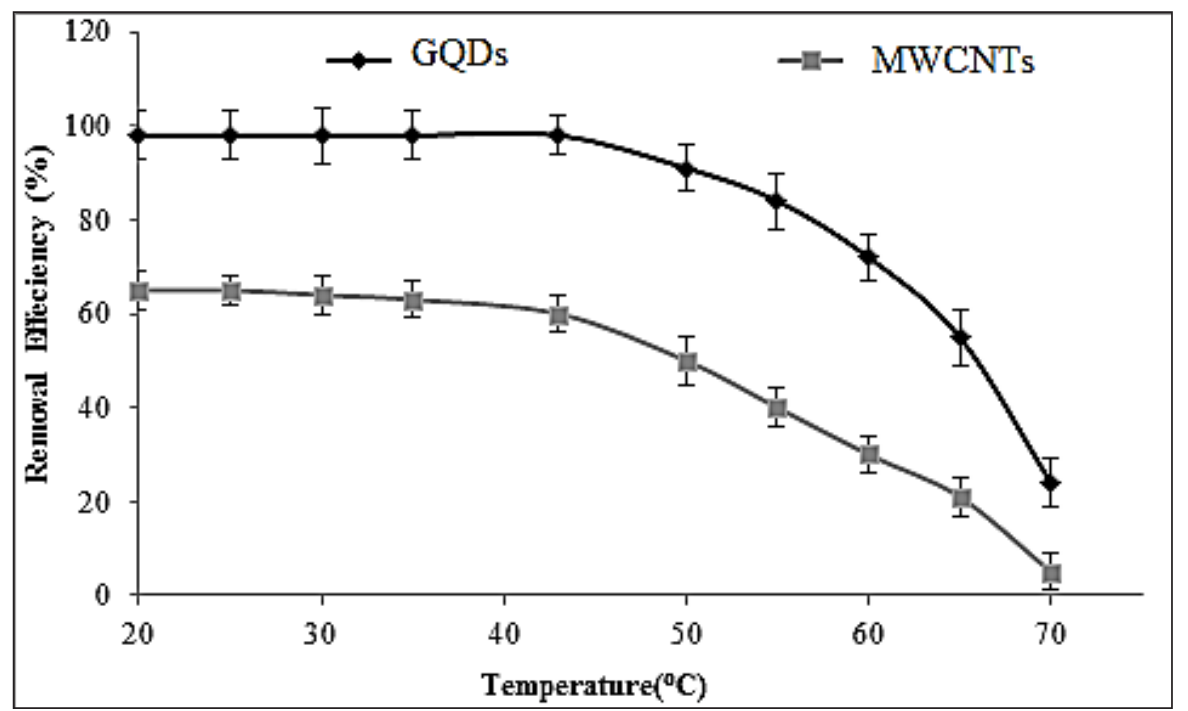

Fig. 6. The effect of temperature on ethylbenzene removal from air by GQDs 


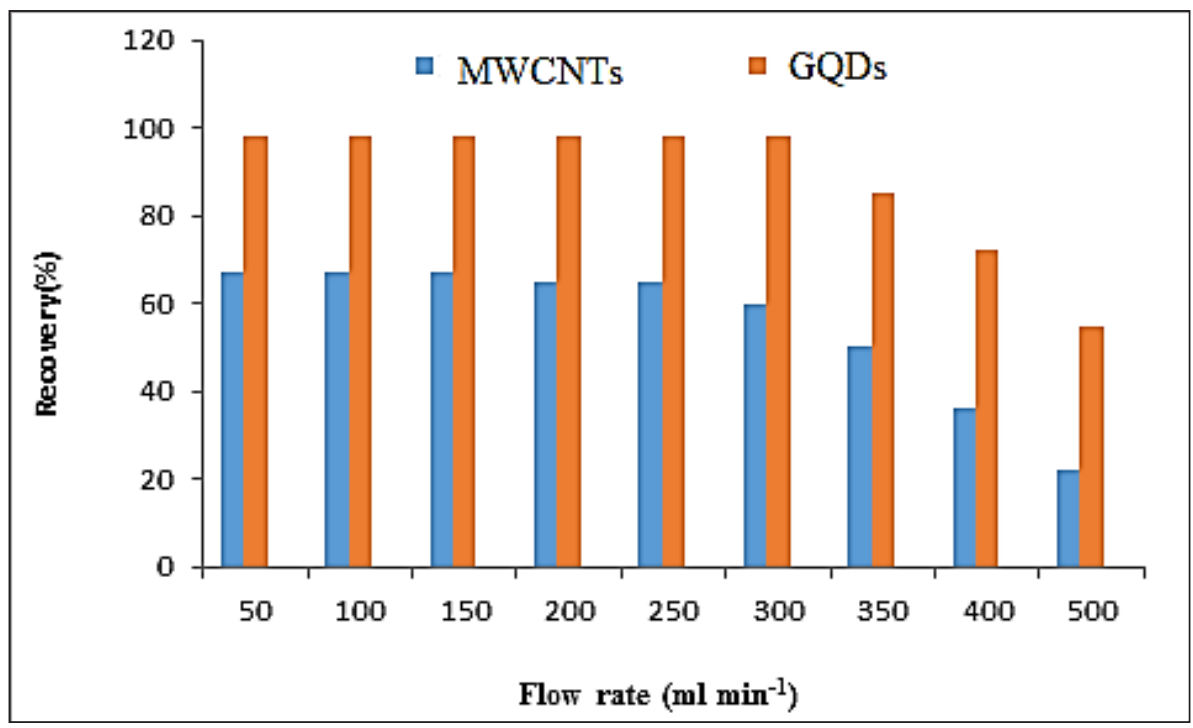

Fig.7 . The effect of flow rate on ethylbenzene removal from air by GQDs

further study.

\subsection{Analyzing and Validation}

The GQDs was selected as a novel sorbent for removal of ethylbenzene vapor from air in present of UV radiation by SGR method. By procedure, a mixture of 1-100 ppm of ethylbenzene in air which was generated in chamber was validated by GC-MS and then, passed through GQDs. After absorption ethylbenzene on GQDs at room temperature, the ethylbenzene desorbed from it at $146^{\circ} \mathrm{C}$ and determined by GC-FID. Since, the standard reference material (SRM) for ethylbenzene in air aren't available, the standard ethylbenzene concentration was generated in a bag $(5 \mathrm{Li})$ by chamber and used for validation by spiking of real samples (Table 3).

\subsection{Discussion}

Fei Yu et al. investigated the removal of TEX from aqueous system by the functionalized magnetic nanoparticle-carbon nanotubes composites that were synthesized, characterized and applied. The APCNTs-KOH composites exhibited high adsorption capacity for TEX onto APCNTs-KOH in a decrease order of ethylbenzene $>$ m-xylene $>$ o-xylene $>$ p-xylene $>$ toluene $(227.05,138.04$, $63.34,249.44$, and $105.59 \mathrm{mg} \mathrm{g}^{-1}$ ), which was higher than current study [40]. In another research,

Table 3. Validation of methodology with GC-FID/SGR for ethylbenzene removal from air by UV-GQDs (ppm)

\begin{tabular}{lccc}
\hline${ }^{*}$ Bag GC-MS & Added & Ethylbenzene & UV-GQDs \\
\hline $1.38 \pm 0.08$ & ----- & $1.34 \pm 0.09$ & Recovery (\%) \\
\hline $5.58 \pm 0.31$ & 1.0 & $2.32 \pm 0.12$ & 97.1 \\
& ----- & $5.51 \pm 0.32$ & 98.0 \\
\hline $10.43 \pm 0.44$ & 5.0 & $10.38 \pm 0.47$ & 98.7 \\
& ----- & $10.07 \pm 0.52$ & 97.4 \\
\hline $20.65 \pm 1.02$ & 10.0 & $19.96 \pm 0.93$ & 96.5 \\
& ----- & $19.89 \pm 1.13$ & 98.9 \\
\hline $80.48 \pm 3.88$ & 20.0 & $40.11 \pm 2.15$ & 96.3 \\
& ----- & $78.65 \pm 4.23$ & 101.1 \\
\hline
\end{tabular}

${ }^{a}$ Mean of three determinations \pm confidence interval $(\mathrm{P}=0.95, \mathrm{n}=5)$

* (Air bag; 1-80 ppm in $5 \mathrm{Li} \mathrm{bag,} 300 \mathrm{~mL} \mathrm{~min}^{-1}$ air flow rate, Peak Area, $25 \mathrm{mg}, \mathrm{T}=25^{\circ} \mathrm{C}$ ) 
Natarajan et al. used the biofiltration method for the removal of the ethylbenzene-xylene mixture while the total inlet loading rate range was 25.408 $\mathrm{g} \mathrm{m}^{-3}$ per hour. The maximum removal capacities attained for ethylbenzene and toluene were 85.63 and $63.2 \mathrm{~g} \mathrm{~m}^{-3}$ per hour respectively, which was lower than our proposed method. The elimination capacities were evaluated at different loading rates and found to vary in a linear pattern. Based on result removal capacities was lower than this study [41]. Ye and Ariya used $\mathrm{Fe}_{3} \mathrm{O}_{4}$ nanoparticles (NPs) at different relative humidities $(\mathrm{RH})$ as adsorption for removal of gaseous benzene, toluene, ethylbenzene and m-xylene (BTEX) and sulfur dioxide $\left(\mathrm{SO}_{2}\right)$. $\mathrm{X}$-ray diffraction, Brunauer-Emmett-Teller, and transmission electron microscopy were deployed for nanoparticle surface characterization. Using gas chromatography equipped with flame ionization detection, Adsorption experiments of BTEX on NPs were measured, which under dry conditions indicated high removal efficiencies (up to $(95 \pm 2) \%$, which are similar to our result [42]. Bina et al. used multi-walled, single-walled, and hybrid carbon nanotubes (MWCNTs, SWCNTs, and HCNTs) for removal of ethylbenzene (EB) from aqueous solution. Ethylbenzene has a higher adsorption tendency on CNTs so that more than $98 \%$ of it adsorbed in the first $14 \mathrm{~min}$, which is related to the low water solubility and the high molecular weight. Isotherm's study indicates that the BET isotherm expression provides the best fit for ethylbenzene sorption by SWCNTs [43]. Kamaei et al. used nitrogen-doped commercial $\mathrm{TiO} 2$ nano-catalysts for photocatalytic decomposition of ethylbenzene in the air using a packed-bed annular photoreactor. The removal efficiency of ethylbenzene under UV irradiation using N-doped catalyst was more than $90 \%$ for the initial concentrations up to $0.586 \mathrm{gm}^{-3}$ (135 ppm) at 1 min residence time Moreover the removal efficiency under visible light radiation could be obtained for the initial concentrations up to 0.1 $\mathrm{gm}^{-3}$ (about $25 \mathrm{ppm}$ ) at $3 \mathrm{~min}$ residence time, which is lower than this article[44]. Hadi et al. used nano-magnetic particles $\left(\mathrm{Fe}_{3} \mathrm{O}_{4}\right)$ as an adsorbent to eliminate ethylbenzene from aqueous solutions.

The characterization of the adsorbent was investigated by transmission electronic microscope (TEM) and X-ray diffraction (XRD) pattern. The results showed that the most amounts of ethylbenzene adsorption and distribution ratio in optimum condition were $49.9 \mathrm{mg} \mathrm{g}^{-1}$ (which was lower than our method) and, $261.9 \mathrm{Lg}^{-1}$ respectively. The results explained that the removal rate of ethylbenzene was higher in batch $(99.8$ $\%)$ rather than continuous (97.4\%) conditions [8]. Ahmed et al. used nZVI for eliminating benzene, toluene, ethylbenzene, and xylene (BTEX) contaminants from aqueous solutions. X-ray diffraction (XRD), UV spectrophotometry, and scanning electron microscopy (SEM) were used for nZVI characterization. The effects of contact time, initial BTEX mixture concentration, adsorbent dose, temperature, and $\mathrm{pH}$ on the amount of BTEX absorbed were studied. The highest removal efficiency of $97 \%$ for the BTEX mixture was achieved at a stirring rate of $100 \mathrm{rpm}$, the temperature of $60^{\circ} \mathrm{C}$, and $\mathrm{pH}$, which is higher than our study. The minimum effective time for efficient removal was $30 \mathrm{~min}$, while the effective dose for BTEX compounds removal was 0.22 $\mathrm{gL}^{-1}[45]$. Yan et al. used $\mathrm{CuMgFe}$ layered double hydroxide (CuMgFe-LDH), for the degradation of ethylbenzene. the degradation efficiency of $0.08 \mathrm{mmol} \mathrm{L}^{-1}$ ethylbenzene was $93.7 \%$ under the optimized conditions at $0.2 \mathrm{~g} \mathrm{~L}^{-1}, \mathrm{CuMgFe}-$ $\mathrm{LDH}$ and $4.0 \mathrm{mmol} \mathrm{L}^{-1}$ persulfate at $\mathrm{pH} \mathrm{7.6}$, which is lower than our result [46]. Azizi et al. used the graphene oxide grafted with polymethyl vinyl ketone and aniline (GO-MVK-ANI), for the elimination of ethylbenzene. The synthesized material was characterized via FTIR, SEM, energydispersive X-ray spectroscopy and BrunauerEmmett-Teller analysis.

Based on the result with initial ethylbenzene concentration of $20 \mathrm{mg} \mathrm{g}^{-1}$ under the optimum 
conditions (the contact time of $11 \mathrm{~min}, \mathrm{pH}$ of 5.64 and adsorbent dose of $3.75 \mathrm{~g} \mathrm{~L}^{-1}$ ), ethylbenzene could be adsorbed (73\%), which is lower than our result [47]. Samarghandi et al. investigated Catalytic Ozonation Process (COP) to treat polluted air streams containing ethylbenzene. Respectively at $50 \mathrm{ppm}$ of this pollutant, for single ozonation and single modified pumice, the best removal efficiency of ethylbenzene was $58-80 \%$, while the maximum removal efficiency of ethylbenzene was $90 \%$ for COP $\left(6 \mathrm{~L} \mathrm{~min}^{-1}\right.$ of flow rate of inlet air, $15 \mathrm{~g}$ of the adsorbent, and $50 \mathrm{ppm}$ of ethylbenzene), which is lower than this study[48]. Also samarghandi et al. used ozone and carbosieve in the catalytic removal of ethylbenzene from the polluted airstream. GC FID was used for sampling and analysis. The results of this study showed that the removal effectiveness of a single ozonation process is averagely less than $25 \%$. Also, whit the concentration increase of ethylbenzene the efficiency of absorbent decreased. The increase ratio of the efficiency in the catalytic ozonation process to the efficiency of carbosieve adsorbent was averagely $45 \%$ which is lower than the current study[49].

\section{Conclusions}

In this study, the GQDs and MWCNTs as nano sorbents were used for ethylbenzene removal from air in present of UV-radiation by SGR method. According to experimental procedure, the simple, reliable and sensitive method based on GQDs was demonstrated in real samples. In optimized conditions, the concentration of ethylbenzene, air flow rate, amount of GQDs and MWCNTs, temperature, and humidity were studied. The results showed, the flow rate $\left(300 \mathrm{ml} \mathrm{L}^{-1}\right)$ can more effected on capacity adsorption by GQDs as physical adsorption. However, in optimized conditions, the removal efficiency and adsorption capacity of GQDs were obtained more than 95\% and $186.4 \mathrm{mg} \mathrm{g}^{-1}$, respectively as compared to MWCNTs.

\section{Acknowledgments}

We are thankful to Kerman University of Medical Sciences (KUMS) and Iranian Petroleum Industry Health Research Institute (IPIHRI).

\section{Reference}

[1] R.K. Nath, M.F.M. Zain, M. Jamil, An environmentfriendly solution for indoor air purification by using renewable photocatalysts in concrete: A review, Renew. Sust. Energ. Rev., 62 (2016) 1184-1194.

[2] J. Yan, Y. Chen, W. Gao, Y. Chen, L. Qian, L. Han, M. Chen, Catalysis of hydrogen peroxide with $\mathrm{Cu}$ layered double hydrotalcite for the degradation of ethylbenzene, Chem., 225 (2019) 157-165.

[3] M. Li, Z. Huang, F. Kang, Progress of volatile organic compounds control technology, Chem. Ind. Eng., 32 (2015) 2-9.

[4] H. Wang, Z. Xiang, L. Wang, S. Jing, S. Lou, S. Tao, J. Liu, M. Yu, L. Li, L. Lin, Emissions of volatile organic compounds (VOCs) from cooking and their speciation: A case study for Shanghai with implications for China, Sci. Total Environ., 621 (2018) 1300-1309.

[5] M. Feiz-Arefi, F. Ghorbani-Shahna, A. Bahrami, H. Ebrahimi, A. Mahjub, Photocatalytic Removal of Methylbenzene Vapors by $\mathrm{MnO} 2 / \mathrm{A} 12 \mathrm{O} 3 / \mathrm{Fe} 2 \mathrm{O} 3$ Nano composite, Iran. J. Health Safe. Environ., 6 (2019) 1158-1166.

[6] S. Zhang, J. You, C. Kennes, Z. Cheng, J. Ye, D. Chen, J. Chen, L. Wang, Current advances of VOCs degradation by bioelectrochemical systems: a review, Chem. Eng. J., 334 (2018) 2625-2637.

[7] A.N. Baghani, A. Sorooshian, M. Heydari, R. Sheikhi, S. Golbaz, Q. Ashournejad, M. Kermani, F. Golkhorshidi, A. Barkhordari, A.J. Jafari, A case study of BTEX characteristics and health effects by major point sources of pollution during winter in Iran, Environ. pollut., 247 (2019) 607-617.

[8] M. Hadei, M. Aalipour, N. Mengli Zadeh, H. Pourzamani, Ethylbenzene removal from aqueous solutions by nano magnetic particles, Arch. Hyg. Sci., 5 (2016) 22-32. 
[9] A. Masih, A.S. Lall, A. Taneja, R. Singhvi, Exposure levels and health risk assessment of ambient BTX at urban and rural environments of a terai region of northern India, Environ. Pollut., 242 (2018) 16781683.

[10] L. Wang, C. Yang, Y. Cheng, J. Huang, H. Yang, G. Zeng, L. Lu, S. He, Enhanced removal of ethylbenzene from gas streams in biotrickling filters by Tween-20 and Zn (II), J. Environ. Sci., 26 (2014) 2500-2507.

[11] A. Carvajal, I. Akmirza, D. Navia, R. Pérez, R. Muñoz, R. Lebrero, Anoxic denitrification of BTEX: Biodegradation kinetics and pollutant interactions, J. environ. manage., 214 (2018) 125 136.

[12] M.H. El-Naas, J.A. Acio, A.E. El Telib, Aerobic biodegradation of BTEX: progresses and prospects, J. Environ. Chem. Eng., 2 (2014) 1104-1122.

[13] R. Moolla, C.J. Curtis, J. Knight, Assessment of occupational exposure to BTEX compounds at a bus diesel-refueling bay: A case study in Johannesburg, South Africa, Sci. Environ., 537 (2015) 51-57.

[14] H.Z. Mousavi, A. Asghari, H. Shirkhanloo, Determination of $\mathrm{Hg}$ in water and wastewater samples by CV-AAS following on-line preconcentration with silver trap, J. Anal. Chem., 65 (2010) 935-939.

[15] H. Shirkhanloo, A. Khaligh, H.Z. Mousavi, M.M. Eskandari, A.A. Miran-Beigi, Ultra-trace arsenic and mercury speciation and determination in blood samples by ionic liquid-based dispersive liquid-liquid microextraction combined with flow injection-hydride generation/cold vapor atomic absorption spectroscopy, Chem. Paper., 69 (2015) 779-790.

[16] H. Shirkhanloo, M. Osanloo, M. Ghazaghi, H. Hassani, Validation of a new and cost-effective method for mercury vapor removal based on silver nanoparticles coating on micro glassy balls, Atmos. Pollut. Res., 8 (2017) 359-365.

[17] L. Zhao, X. Qin, X. Hou, Y. Li, K. Zhang, W. Gong, J. Nie, T. Wang, Research on determination of BTEX in human whole blood using purge and trap- gas chromatography-mass spectrometry combined with isotope internal standard, Microchem. J., 145 (2019) 308-312.

[18] Ethylbenzene, International Agency for Research on Cancer (IARC), 77 (2000) 227-266.

[19] Threshold limit values for chemical substances and physical agents and biological exposure indices, in, American Conference of Governmental Industrial Hygienists(ACGIH), 1995.

[20] S. Wilbur, S. Bosch, Interaction profile for: benzene, toluene, ethylbenzene, and xylenes (BTEX), Agency for Toxic Substances \& Disease Registry (ATSDR), 2004.

[21] M. Delikhoon, M. Fazlzadeh, A. Sorooshian, A.N. Baghani, M. Golaki, Q. Ashournejad, A. Barkhordari, Characteristics and health effects of formaldehyde and acetaldehyde in an urban area in Iran, Environ. pollut., 242 (2018) 938-951.

[22] F. Golkhorshidi, A. Sorooshian, A.J. Jafari, A.N. Baghani, M. Kermani, R.R. Kalantary, Q. Ashournejad, M. Delikhoon, On the nature and health impacts of BTEX in a populated middle eastern city: Tehran, Iran, Atmos. Pollut. Res., 10 (2019) 921-930.

[23] S. Hazrati, R. Rostami, M. Fazlzadeh, F. Pourfarzi, Benzene, toluene, ethylbenzene and xylene concentrations in atmospheric ambient air of gasoline and $\mathrm{CNG}$ refueling stations, Air Qual. Atmos. Health, 9 (2016) 403-409.

[24] M. Bahri, F. Haghighat, Plasma-based indoor air cleaning technologies: the state of the art-Review, Clean Soil Air Water, 42 (2014) 1667-1680.

[25] M. Sansotera, S.G.M. Kheyli, A. Baggioli, C.L. Bianchi, M.P. Pedeferri, M.V. Diamanti, W. Navarrini, Absorption and photocatalytic degradation of VOCs by perfluorinated ionomeric coating with $\mathrm{TiO} 2$ nanopowders for air purification, Chem. Eng. J., 361 (2019) 885-896.

[26] K.W. Shah, W. Li, A Review on Catalytic Nanomaterials for Volatile Organic Compounds VOC Removal and Their Applications for Healthy Buildings, Nanomaterials., 9 (2019) 910.

[27] C. Yang, G. Miao, Y. Pi, Q. Xia, J. Wu, Z. Li, J. 
Xiao, Abatement of various types of VOCs by adsorption/catalytic oxidation: A review, Chem. Eng. J., 370 (2019) 1128-1153.

[28] L. Zhong, F. Haghighat, Photocatalytic air cleaners and materials technologies-Abilities and limitations, Buid. Environ., 91 (2015) 191-203.

[29] M. Malayeri, F. Haghighat, C.-S. Lee, Modeling of volatile organic compounds degradation by photocatalytic oxidation reactor in indoor air: A review, Buid. Environ., 154 (2019) 309-323.

[30] A.H. Mamaghani, F. Haghighat, C.-S. Lee, Photocatalytic oxidation technology for indoor environment air purification: the state-of-the-art, App. Catal. B: Environ., 203 (2017) 247-269.

[31] M. Hussain, P. Akhter, J. Iqbal, Z. Ali, W. Yang, N. Shehzad, K. Majeed, R. Sheikh, N. Russo, VOCs photocatalytic abatement using nanostructured titania-silica catalysts, J. Environ. Chem. Eng., 5 (2017) 3100-3107.

[32] C.H.A. Tsang, K. Li, Y. Zeng, W. Zhao, T. Zhang, Y. Zhan, R. Xie, D.Y. Leung, H. Huang, Titanium oxide based photocatalytic materials development and their role of in the air pollutants degradation: Overview and forecast, Environ. int., 125 (2019) 200-228.

[33] M. Hu, Z. Yao, X. Wang, Graphene-based nanomaterials for catalysis, Ind. Eng. Chem. Res., 56 (2017) 3477-3502.

[34] Y. Pan, X. Yuan, L. Jiang, H. Yu, J. Zhang, H. Wang, R. Guan, G. Zeng, Recent advances in synthesis, modification and photocatalytic applications of micro/nano-structured zinc indium sulfide, Chem. Eng. J., 354 (2018) 407-431.

[35] A. Truppi, F. Petronella, T. Placido, M. Striccoli, A. Agostiano, M.L. Curri, R. Comparelli, Visiblelight-active $\mathrm{TiO} 2$-based hybrid nanocatalysts for environmental applications, Catal., 7 (2017) 100.

[36] A. Rashidi, M. Akbarnejad, A. Khodadadi, Y. Mortazavi, A. Ahmadpourd, Single-wall carbon nanotubes synthesized using organic additives to Co-Mo catalysts supported on nanoporous $\mathrm{MgO}$, Nanotech., 18 (2007) 315605.

[37] Y. Xu, H. Bai, G. Lu, C. Li, G. Shi, Flexible graphene films via the filtration of water-soluble noncovalent functionalized graphene sheets, J. Am. Chem. Soc., 130 (2008) 5856-5857.

[38] N.I. Kovtyukhova, P.J. Ollivier, B.R. Martin, T.E. Mallouk, S.A. Chizhik, E.V. Buzaneva, A.D. Gorchinskiy, Layer-by-layer assembly of ultrathin composite films from micron-sized graphite oxide sheets and polycations, Chem. mater., 11 (1999) 771-778.

[39] Y. Dong, H. Pang, S. Ren, C. Chen, Y. Chi, T. Yu, Etching single-wall carbon nanotubes into green and yellow single-layer graphene quantum dots, Carbon, 64 (2013) 245-251.

[40] F. Yu, J. Ma, J. Wang, M. Zhang, J. Zheng, Magnetic iron oxide nanoparticles functionalized multiwalled carbon nanotubes for toluene, ethylbenzene and xylene removal from aqueous solution, Chemosphere, 146 (2016) 162-172.

[41] R. Natarajan, J. Al-Sinani, S. Viswanthan, R. Manivasagan, Biodegradation of ethyl benzene and xylene contaminated air in an up flow mixed culture biofilter, Int. biodet. biodeg., 119 (2017): 309-315.

[42] Z.Y. Connie, P.A. Ariya, Co-adsorption of gaseous benzene, toluene, ethylbenzene, m-xylene (BTEX) and $\mathrm{SO} 2$ on recyclable $\mathrm{Fe} 3 \mathrm{O} 4$ nanoparticles at 0-101\% relative humidities, J. Environ. Sci., 31 (2015) 164-174.

[43] B. Bina, H. Pourzamani, A. Rashidi, M.M. Amin, Ethylbenzene removal by carbon nanotubes from aqueous solution, J. environ. pub. health, 2012 (2012).

[44] M. Kamaei, H. Rashedi, S.M.M. Dastgheib, S. Tasharrofi, Comparing photocatalytic degradation of gaseous ethylbenzene using $\mathrm{N}$-doped and pure $\mathrm{TiO} 2$ nano-catalysts coated on glass beads under both UV and visible light irradiation, Catal., 8 (2018) 466.

[45] A.S. Mahmoud, M.K. Mostafa, S.A. Abdel-Gawad, Artificial intelligence for the removal of benzene, toluene, ethyl benzene and xylene (BTEX) from aqueous solutions using iron nanoparticles, Water Suply., 18 (2018) 1650-1663.

[46] J. Yan, Y. Chen, L. Qian, W. Gao, D. Ouyang, 
M. Chen., Heterogeneously catalyzed persulfate with a $\mathrm{CuMgFe}$ layered double hydroxide for the degradation of ethylbenzene. J. hazard. mater., 338 (2017) 372-380.

[47] A. Azizi, A. Torabian, E. Moniri, A.H. Hassani, H. Ahmad Panahi, Investigating the removal of ethylbenzene from aqueous solutions using modified graphene oxide: application of response surface methodology, Int. J. Environ. Sci. Tec., 15 (2018) 2669-2678.

[48] M.R. Samarghandi, Z. Daraee, G. Shekher, G. Asgari, A. Reza Rahmani, A. Poormohammadi., Catalytic ozonation of ethyl benzene using modified pumice with magnesium nitrate from polluted air. Int. J. Environ. Stutdies, 74 (2017), 486-499.

[49] M.R. Samarghandi, G. Asgari, F. Ghorbani, S.A. Babaee., The study of effect the combined use of ozone and carbosieve in the catalytic removal of ethylbenzene from the polluted airstream, J. Neyshabur UN. Medical. Sci., 5 (2017) 86-98. 